\title{
çência
}

\section{AVALIAÇÃO DA ASSISTÊNCIA À PESSOA IDOSA NA ATENÇÃO PRIMÁRIA À SAÚDE: PERSPECTIVA DE USUÁRIOS}

\section{Evaluation of assistance to the elderly person in Primary Health Care: users perspective}

Ana Mayara Gomes de Souza • Enfermeira. Mestranda em Saúde Coletiva pelo Programa de Pós-Graduação em Saúde Coletiva (PPGSCol) da Universidade Federal do Rio Grande do Norte (UFRN). E-mail: ana.mayara@yahoo.com.br

Tainara Lôrena dos Santos Ferreira • Enfermeira. Mestranda em Saúde Coletiva pelo Programa de PósGraduação em Saúde Coletiva (PPGSCol) da Universidade Federal do Rio Grande do Norte (UFRN). E-mail: tainara_lorena@hotmail.com

Katarina Marcia Rodrigues dos Santos • Acadêmica do Curso de Enfermagem da Faculdade de Ciências da Saúde do Trairi (FACISA/UFRN). Bolsista de Iniciação Científica PIBIC CNPq-UFRN. E-mail: katarinamrsantos@hotmail.com

Débora Joyce Duarte de Oliveira • Acadêmica do Curso de Enfermagem da Faculdade de Ciências da Saúde do Trairi (FACISA/UFRN). Bolsista de Iniciação Científica PIBIC CNPq-UFRN. E-mail: jooyceduarte@hotmail.com

Fábia Barbosa de Andrade • Enfermeira. Doutora em Ciências da Saúde. Professora Adjunto III da UFRN. E-mail: fabiabarbosabr@yahoo.com.br

Autora responsável pela correspondência:

Ana Mayara Gomes de Souza. Enfermeira. Mestranda em Saúde Coletiva pelo Programa de Pós-Graduação em Saúde Coletiva (PPGSCol) da Universidade Federal do Rio Grande do Norte (UFRN).

E-mail: ana.mayara@yahoo.com.br

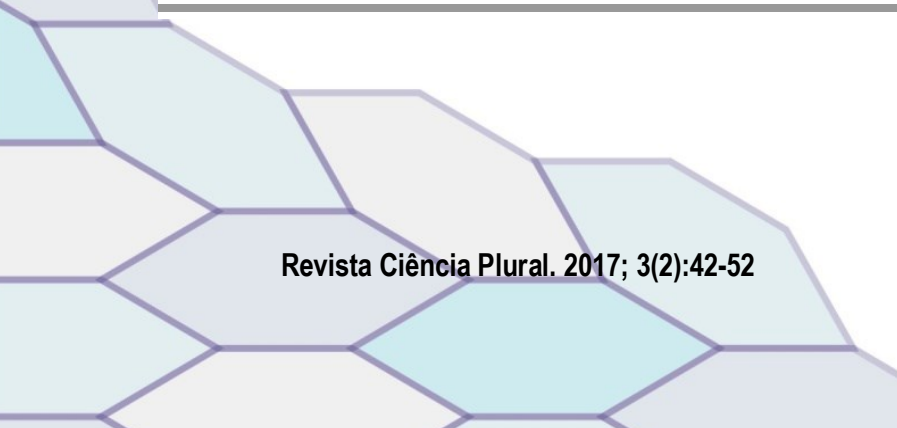




\section{ciência plural}

RESUMO

Introdução: O aumento da expectativa de vida é de suma importância no contexto social e também um dos nossos grandes desafios; de modo que se tornam fundamentais, estudos que priorizem a avaliação da atenção à população idosa. Objetivo: Avaliar, a partir da percepção dos usuários idosos, a qualidade da assistência da equipe profissional na Atenção Primária à Saúde da pessoa idosa. Metodologia: Trata-se de um estudo exploratório descritivo com abordagem quantitativa realizada na cidade de Santa Cruz, estado Rio Grande do Norte, Brasil, utilizando-se uma amostra de 130 idosos. Resultados: No que se refere à qualidade do atendimento na APS, podemos observar que 48,5\% dos usuários afirmou ser bom, uma vez que $26,2 \%$ verbalizaram que raramente havia rotatividade profissional. Em relação à educação em saúde, observou-se que $82,3 \%$ nunca receberam orientações sobre prevenção do câncer e controle de quedas e $41,5 \%$ algumas vezes recebiam sobre importância de realização de atividade física. No que diz respeito à necessidade de encaminhamento dos pacientes à outros serviços observou-se que 50,0\% algumas vezes foram encaminhados e $29,2 \%$ dizem algumas vezes terem sido contra referenciado. Conclusão: A atuação dos profissionais da APS foi adequada, o que confere boa qualidade aos serviços. As fragilidades ainda existentes, oferecem subsídios para que estes possam ser estimulados a elaborar novas estratégias para melhorias à saúde do idoso.

Palavras-chave: Saúde do Idoso; Atenção Primária à Saúde; Pesquisa sobre Serviços de Saúde.

\section{ABSTRACT}

Introduction: The increase in life expectancy is of paramount importance in the social context and also one of our great challenges; thus, studies that prioritize the evaluation of attention to the elderly population become fundamental. Objective: To evaluate, from the perception of the elderly users, the quality of the assistance of the professional team in the Primary Health Care to the elderly person. Methodology: This is a descriptive exploratory study with a quantitative approach performed in the city of Santa Cruz, Rio Grande do Norte State, Brazil, using a sample of 130 elderly people. Results: Regarding the quality of care in PHC, we can observe that $48.5 \%$ of the users said that they were good, since $26.2 \%$ stated that there was rarely any professional turnover. Regarding health education, it was observed that $82.3 \%$ never received guidance on cancer prevention and control of falls and $41.5 \%$ were sometimes given over importance of physical activity. Regarding the need to refer patients to other services, it was observed that $50.0 \%$ were sometimes referred and $29.2 \%$ said they were sometimes referred to as referrals. Conclusion: The performance of PHC professionals was adequate, which gives good quality to the services. The fragilities that still exist, offer subsidies so that they can be stimulated to elaborate new strategies for improvements in the health of the elderly.

Key Words: Health of the Elderly; Primary Health Care; Health Services Research. 


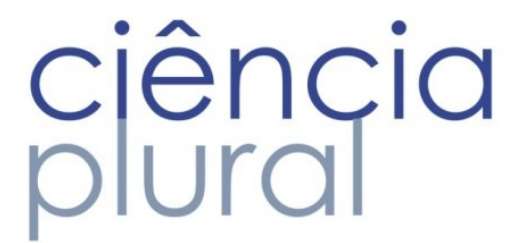

Introdução

A população idosa vem aumentando consideravelmente em todo país. Em virtude disso tornam-se fundamentais, estudos que priorizem uma atenção especial às questões concernentes à esta população na perspectiva de garantir não só longevidade, mas uma vida ativa e saudável sem que a mesma se torne um fardo pesado para sociedade, família e principalmente, para a pessoa idosa.

O envelhecimento da população é um dos maiores triunfos da humanidade e também um dos nossos grandes desafios. Estudos realizados pela Organização Mundial de Saúde ${ }^{1}$ mostram que em todo o mundo, a proporção de pessoas com 60 anos ou mais está crescendo rapidamente, e que entre 1970 e 2025 , espera-se um crescimento de 223,0\%; em 2025, existirá um total de aproximadamente 1,2 bilhões de pessoas com mais de 60 anos.

Para a saúde coletiva, o envelhecimento populacional se apresenta como um dos maiores desafios, mormente nos países em desenvolvimento, onde a pobreza e a desigualdade social ganham destaque 2 . Frente a isso se tornam indispensáveis espaços de promoção à saúde que considerem a pluralidade cultural que emerge das experiências contextuais desse grupo etário.

Atender ao idoso de forma integral é preocupar-se não apenas com os aspectos relacionados ao processo saúde-doença que o mesmo enfrenta. Assim, é necessário atendê-lo em suas necessidades físicas, emocionais, espirituais e sociais, integrando-os com outras pessoas, em especial outros indivíduos da mesma faixa etária que a sua, proporcionando-lhes atividades que os integre a população como um todo ${ }^{3}$.

Atualmente no Brasil, nos deparamos com um processo de envelhecimento populacional e com um modelo de transição epidemiológica singular. Assim é necessário que, além de competência técnicocientífica em todos os níveis da atenção à saúde, haja também um projeto de organização política capaz de abarcar todas as demandas e interfaces desses fenômenos ${ }^{4}$. A Política Nacional da Pessoa Idosa define que a atenção à saúde do idoso tem como porta de entrada a Atenção Primária à Saúde (APS) 5 .

A Estratégia Saúde da Família (ESF) por sua vez configura-se como principal modelo assistencial, tendo por objetivo reorganizar o modelo tradicional de atendimento à saúde, que preconiza uma equipe de caráter multiprofissional que trabalha com definição de território de abrangência, adscrição de clientela, cadastramento e acompanhamento da população residente na área ${ }^{6}$. A unidade de saúde da família além de ser o primeiro nível de atenção, caracteriza-se por integrar uma rede de serviços mais complexos.

Dessa forma, políticas de direitos das pessoas idosas preconizam a criação de programas que aperfeiçoem o potencial da pessoa idosa na manutenção de sua vida social, de seu bem-estar e de um viver 


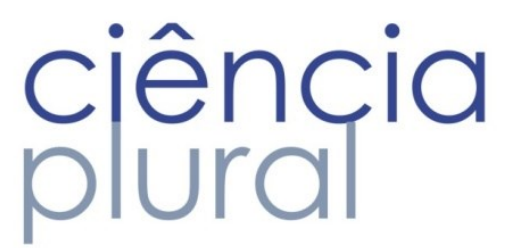

condigno ${ }^{4}$. É oportuna e necessária a capacitação da equipe, visto que atender ao idoso em suas particularidades é diferente de atender qualquer outro grupo etário, em especial, se o idoso estiver institucionalizado, pois além de prestar os cuidados como membro da equipe multiprofissional, o profissional é a pessoa mais próxima do idoso, que fará papel de cuidador, familiar e amigo ${ }^{3}$.

Nesta perspectiva, considerando o aumento da expectativa de vida da população idosa e sua importância no contexto social, bem como a importância de contribuir para manutenção dos direitos assegurados por lei e pelas politicas do Sistema Único de Saúde (SUS), este estudo tem como objetivo avaliar, a partir da percepção dos usuários idosos, a qualidade da assistência da equipe profissional na APS à esse grupo etário.

\section{Metodologia}

Trata-se de um estudo exploratório descritivo com abordagem quantitativa realizada com idosos usuários dos serviços de saúde do município de Santa Cruz, localizado no interior do Rio Grande do Norte, Brasil. O presente estudo é um recorte da pesquisa intitulada Atenção Primária à Saúde: um estudo de avaliação na perspectiva dos usuários.

A escolha da amostra se deu de modo aleatório, ou seja, mediante a um sorteio com base no cadastro da rede de APS do município, respeitando à proporcionalidade dos usuários cadastrados. A pesquisa tratase de uma avaliação da qualidade do atendimento multiprofissional e sua relação quanto à rotatividade dos profissionais nas unidades, as orientações repassadas com foco na prevenção de câncer, quedas e realização de atividades físicas, a qualidade dos registros de saúde e necessidade de encaminhamento a outros serviços.

A amostra calculada total foi de 130 sujeitos e a coleta de dados foi realizada em domicílio excluindo qualquer investigação aprofundada sobre possíveis agravos acometidos aos pacientes entrevistados. 0 critério de inclusão escolhido foi ser idoso, com idade mínima de 60 anos e máxima de 96, possuir capacidade cognitiva preservada, residir na comunidade da Unidade Básica de Saúde (UBS) sorteada e assinar o Termo de Consentimento Livre e Esclarecido (TCLE).

Os dados coletados foram armazenados e analisados no Statistical Package for the Social Sciences (SPSS), versão 17.0. Para análise de dados, obteve-se frequências absoluto-relativas, medidas de tendência central e dispersão. No âmbito da análise bivariada, foram realizados testes qui-quadrado, 


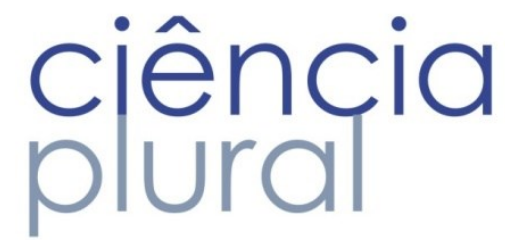

convecionando-se como nível de significância uma probabilidade inferior a 0,05 e Intervalo de Confiança de $95 \%$.

O estudo foi realizado após apreciação do Comitê de Ética em Pesquisa da Universidade Federal do Rio Grande do Norte (UFRN), segundo o que preconiza a Resolução 466/2012 do Conselho Nacional de Saúde ${ }^{7}$ sobre a pesquisa com seres humanos, sob o número de parecer 152/2012.

\section{Resultados}

O presente estudo buscou avaliar a qualidade do atendimento da equipe multiprofissional na APS, tendo como base os princípios do SUS, com foco na promoção, proteção e manutenção da saúde da pessoa idosa.

Para isso, foram entrevistadas 130 pessoas, sendo 92 (70,8\%) mulheres. A média de idades foi de 72,8; obtendo assim mediana de 72,0 e desvio padrão de 8,3. A amostra mostrou-se satisfatória para avaliar a qualidade do atendimento aos idosos na APS.

Tabela 1: Classificação da qualidade da assistência na Atenção Primária à Saúde na ótica dos usuários.

Santa Cruz/RN, 2017

\begin{tabular}{c|c|c|c|c|c|c}
\hline Variável & Péssima & Ruim & Regular & Boa & Excelente & Total \\
\hline $\begin{array}{c}\text { Classificação da } \\
\text { qualidade da }\end{array}$ & $\begin{array}{c}6,2 \% \\
(\mathrm{n}=08)\end{array}$ & $\begin{array}{c}6,9 \% \\
(\mathrm{n}=09)\end{array}$ & $\begin{array}{c}32,3 \% \\
(\mathrm{n}=42)\end{array}$ & $\begin{array}{c}48,5 \% \\
(\mathrm{n}=63)\end{array}$ & $\begin{array}{c}6,2 \% \\
(\mathrm{n}=08)\end{array}$ & $\begin{array}{c}100 \% \\
(\mathrm{n}=130)\end{array}$ \\
$\begin{array}{c}\text { Assistência sob a } \\
\text { ótica do usuário }\end{array}$ & & & & & \\
\hline
\end{tabular}

Quando questionados quanto à qualidade do atendimento na APS, observa-se na tabela 1 que uma totalidade de 48,5\% ( $n=63$ ) dos usuários afirmou ser "bom" seguido de 32,3\% ( $n=42$ ) que consideraram "razoáveis". Ainda, uma parcela de 13,1\% (n=17) considerou a qualidade "ruim ou péssima", seguidos de $6,2 \%(n=08)$ que afirmaram ser "excelente". 
Tabela 2: Associação entre classificação da qualidade do atendimento, a rotatividade do profissional de nível superior nas unidades de saúde e o conhecimento prévio da história clínica do paciente.

Santa Cruz/RN, 2017

\begin{tabular}{c|c|c|c|c|c|c}
\hline Variáveis & Nunca & Raramente & $\begin{array}{c}\text { Algumas } \\
\text { vezes }\end{array}$ & Frequentemente & Sempre & $\mathbf{p}<\mathbf{0 , 0 5}$ \\
\hline $\begin{array}{c}\text { Rotatividade do } \\
\text { profissional }\end{array}$ & $\begin{array}{c}10,8 \% \\
(n=14)\end{array}$ & $\begin{array}{c}26,2 \% \\
(n=34)\end{array}$ & $\begin{array}{c}42,3 \% \\
(n=55)\end{array}$ & $\begin{array}{c}8,5 \% \\
(n=11)\end{array}$ & $\begin{array}{c}12,3 \% \\
(n=16)\end{array}$ & 0,003 \\
\hline $\begin{array}{c}\text { Conhecimento da } \\
\text { História Clínica do } \\
\text { Paciente }\end{array}$ & $\begin{array}{c}30,0 \% \\
(n=39)\end{array}$ & $\begin{array}{c}8,5 \% \\
(n=11)\end{array}$ & $\begin{array}{c}33,8 \% \\
(n=44)\end{array}$ & $\begin{array}{c}9,2 \% \\
(n=12)\end{array}$ & $\begin{array}{c}18,5 \% \\
(n=24)\end{array}$ & 0,000 \\
\hline
\end{tabular}

Relacionado à rotatividade de profissionais de nível superior nas unidades de saúde do município, ou seja, o profissional que o atendeu na primeira consulta já não foi que o da última (tabela 2), 42,3\% ( $n=55$ ) dos usuários afirmou que "algumas vezes" ocorre; $26,2 \%$ ( $n=34)$ verbalizaram que "raramente" há mudanças desses profissionais; 12,3\% ( $n=16)$ afirmam que essas mudanças "sempre" acontecem.

Ainda na tabela 2, quanto à relação entre à qualidade do atendimento e o conhecimento prévio da história clínica do paciente, os usuários observaram que 33,8\% ( $n=44)$ "algumas vezes" estes profissionais conheciam; 30,0\% (n=39) "nunca"; 18,5\% (n=24) "sempre" conhecem. Assim, o teste de qui-quadrado revelou uma associação significativa entre a qualidade do atendimento e a mudança do profissional/conhecimento deste sobre a história clínica do paciente.

Tabela 3: Associação entre classificação da qualidade do atendimento e educação em saúde voltada à prevenção do câncer, prevenção e controle de quedas e realização de atividades físicas.

Santa Cruz/RN, 2017

\begin{tabular}{c|c|c|c|c|c|c}
\hline Variáveis & Nunca & Raramente & $\begin{array}{c}\text { Algumas } \\
\text { vezes }\end{array}$ & Frequentemente & Sempre & $p<0,05$ \\
\hline Câncer & $\begin{array}{c}36,9 \% \\
(n=48)\end{array}$ & $\begin{array}{c}6,9 \% \\
(n=09)\end{array}$ & $\begin{array}{c}35,4 \% \\
(n=46)\end{array}$ & $\begin{array}{c}9,2 \% \\
(n=12)\end{array}$ & $\begin{array}{c}11,5 \% \\
(n=15)\end{array}$ & 0,000 \\
\hline Quedas & $45,4 \%$ & $5,4 \%$ & $30,0 \%$ & $10,8 \%$ & $8,5 \%$ & 0,001 \\
& $(n=59)$ & $(n=07)$ & $(n=39)$ & $(n=14)$ & $(n=11)$ & \\
\hline Atividades & $15,4 \%$ & $6,9 \%$ & $41,5 \%$ & 13,1 & 23,1 & 0,004 \\
físicas & $(n=20)$ & $(n=09)$ & $(n=54)$ & $(n=17)$ & $(n=30)$ & \\
\hline
\end{tabular}

A Tabela 3 faz referência ao recebimento de orientações, ou seja, ações voltadas para educação em saúde abordando a temática da prevenção do câncer, prevenção e controle de quedas e realização de atividades físicas, realizadas pela equipe multiprofissional do serviço de saúde. Com relação à prevenção do câncer, observou-se que 36,9\% (n=48) afirmaram que "nunca" tiveram orientações; 35,4\% (n=46) 


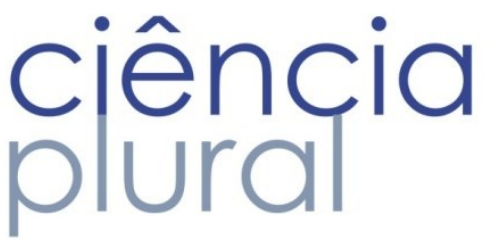

"raramente"; 11,5\% ( $n=15)$ verbalizaram que "sempre", controle e prevenção de quedas 45,4\% (n=59) relataram que "nunca" foram orientados sobre; 30,0\% (n=39) "algumas vezes"; 10,8\% ( $n=14)$ afirmaram que "frequentemente". Por fim, quanto à importância de realização de atividade física observou-se que 41,5\% $(n=54)$ atestaram que "algumas vezes" isso acontecia durante 0 atendimento; $23,1 \%(n=30)$ "sempre"; 15,4\% $(n=20)$ afirmaram que "nunca".

Tabela 4: Associação entre classificação da qualidade do atendimento, a necessidade de encaminhamento a outros serviços de saúde e a contra referência dos serviços a partir dos registros e boletins de saúde.

Santa Cruz/RN, 2017

\begin{tabular}{|c|c|c|c|c|c|c|}
\hline Variáveis & Nunca & Raramente & $\begin{array}{c}\text { Algumas } \\
\text { vezes }\end{array}$ & Frequentemente & Sempre & $p<0,05$ \\
\hline $\begin{array}{l}\text { Necessidade de outros } \\
\text { serviços para } \\
\text { realização de } \\
\text { consultas e/ou exames }\end{array}$ & $\begin{array}{l}14,6 \% \\
(n=19)\end{array}$ & $\begin{array}{l}7,7 \% \\
(n=10)\end{array}$ & $\begin{array}{l}50,0 \% \\
(n=65)\end{array}$ & $\begin{array}{l}15,4 \% \\
(n=20)\end{array}$ & $\begin{array}{l}12,3 \% \\
(n=16)\end{array}$ & 0,000 \\
\hline $\begin{array}{c}\text { Contra referência } \\
\text { serviço/profissional de } \\
\text { saúde }\end{array}$ & $\begin{array}{l}23,8 \% \\
(n=31)\end{array}$ & $\begin{array}{c}7,7 \% \\
(n=10)\end{array}$ & $\begin{array}{l}29,2 \% \\
(n=38)\end{array}$ & $\begin{array}{l}11,5 \% \\
(n=15)\end{array}$ & $\begin{array}{l}27,7 \% \\
(n=36)\end{array}$ & 0,001 \\
\hline
\end{tabular}

No que diz respeito à necessidade de encaminhamento dos pacientes a outros serviços como, serviços de exame ou serviços especializados prestados por outros profissionais, observou-se a partir dos dados da tabela 4 que 50,0\% ( $n=65)$ que "algumas vezes" foram encaminhados; $15,4 \% \quad(n=20)$ "frequentemente"; 14,6\% (n=19) verbalizaram que "nunca". Com relação à contra referencia destes serviços a partir dos boletins/registros de saúde preenchidos por seus profissionais, 29,2\% ( $n=38$ ) afirmaram que "algumas vezes" havia; $27,7 \%(n=36)$ "sempre"; $23,8 \%(n=31)$ atestaram que "nunca".

\section{Discussão}

A saúde deve ser vista a partir de uma perspectiva ampla, resultado de um trabalho intersetorial e transdisciplinar de promoção de modos de vida saudável em todas as idades. abe aos profissionais da saúde liderar os desafios do envelhecimento saudável para que os idosos sejam um recurso cada vez mais valioso para suas famílias, comunidades e para o país ${ }^{1}$.

Os resultados mostram associação positiva da qualidade do atendimento na ótica dos usuários e a conduta profissional na APS. Conforme mostra a Tabela 1, existe um propósito claro de fortalecimento da APS, com ênfase na incorporação de valores, princípios e elementos próprios da atenção primária efetiva, o que leva ao avanço em relação ao incentivo de incremento meramente numérico, sem compromisso com a qualidade ${ }^{8}$. 


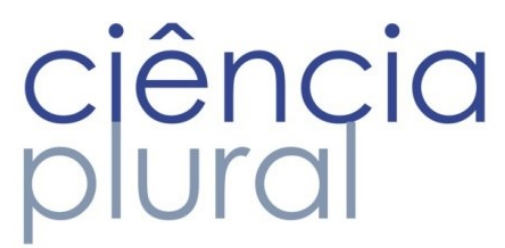

A grande expansão da ESF trouxe a necessidade de qualificação da atenção básica ofertada à população, tornando necessário o desenvolvimento de ferramentas que permitam a implantação de estratégias de forma permanente, para orientar com agilidade o processo decisório, e melhorar os resultados para a população ${ }^{9}$. Desse modo, os usuários devem ser atores principais na avaliação da qualidade da assistência que recebem. Tal comportamento os valoriza enquanto consumidores além do que, sua satisfação pode influenciar significativamente na adesão a terapêutica adotada, sendo importante para 0 fortalecimento da APS.

A relação usuário-profissional se faz primordial, como já foi dito, ela estimula a acessibilidade do usuário e a sua adesão as ações de saúde e terapêutica. A rotatividade de profissionais, descrito na tabela 2, mostrada a partir da porcentagem maior para "algumas vezes" seguido por "raramente" evidência que há a existência de um vínculo, porém, quando nos reportamos ao conhecimento prévio da clínica dos pacientes os dados demostram algumas falhas nesta relação que podem influenciar diretamente na satisfação desse usuário com o serviço.

Em virtude disso, reforça-se a importância da avaliação da continuidade, priorizando a existência de meios de registro de informações eficazes, de modo a permitir que todos os dados sejam corretamente registrados em prontuários. Ademais, o acesso facilitado e acolhimento de qualidade articulam-se e se complementam na implementação de práticas em serviços de saúde, devem ser vistos, portanto, como um dispositivo potente para propiciar vínculo entre equipe e população, trabalhador e usuário, questionar o processo de trabalho, desencadear cuidado integral e modificando assim a clínica ${ }^{8}$.

Outro ponto analisado e apresentado na tabela 3 foi a educação em saúde na prevenção de agravos como câncer, quedas e a estimulação à prática de exercícios físicos, sobre prevenção e controle de quedas e câncer, as respostas com maior significância são "nunca" seguidas por "algumas vezes" com relação a pratica de atividades físicas "algumas vezes" apresenta-se com maior significância.

Percebe-se a necessidade de estimular a autonomia da pessoa idosa e a sua independência, através de ações que os tornem corresponsáveis pela sua saúde, resgatando o autocuidado e inovando hábitos que venham a promover a saúde. 0 autocuidado contribui para que a pessoa idosa melhore o seu convívio familiar e social, ampliando sua autonomia e autoestima com vistas ao envelhecer saudável. Ele cria condições favoráveis que facilitam e incentivam a diminuição da dependência na relação profissional-clientefamilia e a prevenção de sequelas negativas ${ }^{10}$. 


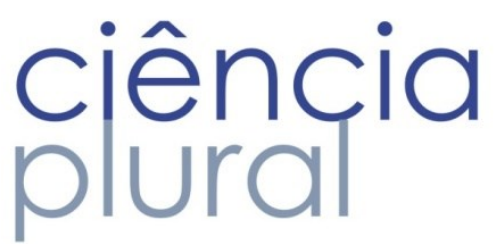

Os profissionais de saúde devem promover atividades preventivas direcionadas à manutenção da funcionalidade, visando postergar o aparecimento de incapacidades que impõem limitações à independência do idoso. 0 idoso deve ser alvo de ações educativas e atendimento individual, de forma que 0 enfermeiro possa estimular 0 autocuidado ${ }^{11}$.

Com relação à prática de exercícios físicos pode-se dizer que a atividade física contribui para a longevidade, mostrando-se importante na medida em que proporciona um aumento na qualidade de vida, auxiliando na manutenção da capacidade funcional e na manutenção plena das habilidades físicas e mentais do idoso' ${ }^{12}$.

De modo específico, programas de atividade física podem contribuir de forma expressiva na qualidade de vida na população de idosos, tanto pelo engajamento social que eles promovem, quanto pelo estímulo positivo nos aspectos físicos, o que na prática resulta em maior autonomia ${ }^{13}$. Mais uma vez, cabe, portanto, ao profissional da área da saúde conscientizar-se e prevenir o processo de envelhecimento por meio de métodos que favoreçam e estimulem a prática constante de exercícios físicos para, assim, proporcionar um envelhecimento saudável2.

Por fim, se faz necessário garantir o acesso ao usuário em todos os níveis de assistência de acordo com as necessidades eco regionais conforme versa a regionalização da assistência. Surge o desafio de trabalhar de forma articulada e com vistas a romper a fragmentação, com profissionais aptos a trabalhar de forma contínua. No estudo a partir da análise da tabela 4, foi possível identificar essas características uma vez que "algumas vezes" foi necessário o encaminhamento dos usuários a outros serviços de maior complexidade, precisando de um maior fortalecimento no tocante da contra referência destes a APS.

A integração da saúde, com constituição de redes regionalizadas e integradas de atenção, é condição indispensável para a qualificação e a continuidade do cuidado, tem grande importância na superação de lacunas assistenciais, além da racionalização e otimização dos recursos assistenciais disponíveis ${ }^{14}$. A implementação da estratégia de saúde da família em grandes centros urbanos difunde uma nova perspectiva para a atenção primária no Brasil, voltada para a família e a comunidade, que tem potencialidades para desencadear mudanças no modelo assistencial que efetivem o direito à saúde no cotidiano dos cidadãos ${ }^{15}$.

A estratégia de saúde da família apresenta-se como forma de prestar uma assistência digna, respeitosa, integral e ética aos usuários. Respeitar aos idosos é respeitá-los em sua integralidade, proporcionando atividades culturais, de lazer e educativas; transporte de um lugar a outro com segurança e 


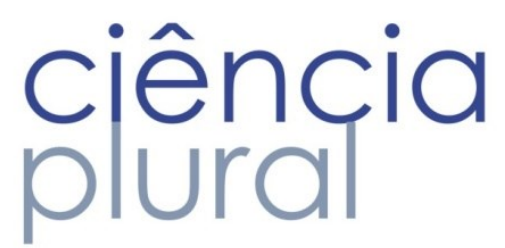

conforto, para que exerçam seu direito à liberdade; desenvolver ambiente de convívio pacífico, assegurando o bem-estar e a efetivação do seu direito à vida 3 .

\section{Conclusões}

A partir dos resultados apresentados e análise das variáveis percebe-se que a atuação dos profissionais da APS foi adequada, o que confere boa qualidade aos serviços, no entanto, ainda foram encontradas algumas lacunas. Faz-se necessário que a equipe profissional amplie as ações voltadas para uma melhor educação em saúde, com oficinas, grupos de conversas e principalmente nas consultas de rotina, cujas estratégias favorecem o vínculo profissional/usuário tornando ativa a assistência.

Ainda foi possível identificar um interesse significativo dos profissionais em encaminhar os usuários à serviços especializados e de maior complexidade. $O$ fato destaca o comprometimento dos profissionais em seguir a hierarquia e tornar efetivo o modelo das Redes de Atenção à saúde (RAS), como preconiza o SUS. Isto é evidenciado quando os usuários respondem que "frequentemente" eram referenciados.

Apesar das limitações no que diz respeito a ser um estudo local, espera-se que este estudo possa favorecer uma maior reflexão e motivação dos profissionais, oferecendo subsídios para que estes possam ser estimulados a elaborar novas estratégias para trazer melhorias à saúde do idoso e assim conseguir uma totalidade na assistência, com foco não apenas na longevidade, mas na promoção, prevenção e proteção da saúde, que associados garantem uma melhor qualidade de vida ao idoso-usuário.

\section{Referências}

1. World Health Organization. Envelhecimento ativo: uma política de saúde. Brasília: Organização PanAmericana da Saúde; 2005. 60 p.

2. Andrade FB, Ferreira Filha MO, Dias MD, Silva AO, Costa ICC, Lima EAR, et al. Promoção da saúde mental do idoso na atenção básica: as contribuições da terapia comunitária. Texto Contexto Enferm, 2010 Jan-Mar; 19(1): 129-36.

3. Martins MS, Massarollo MCKB. Mudanças na assistência ao idoso após promulgação do Estatuto do Idoso segundo profissionais de hospital geriátrico. Rev Esc Enferm USP, 2008; 42(1): 26-33.

4. Alvarez AM, Gonçalves LHT. Enfermagem e o cuidado ao idoso no domicilio. Rev. bras. enferm. Brasília, set-out. 2012; 65(5): 715-6.

5. Ministério da Saúde (Brasil). Portaria 2528/GM, de 19 de outubro de 2006. Aprova a Política Nacional de Saúde da Pessoa Idosa. Brasília: Ministério da Saúde; 2006.

6. Garcia VA, Vendruscolo C, Tesser CD, Argenta CE. Concepções de promoção da saúde que permeiam 


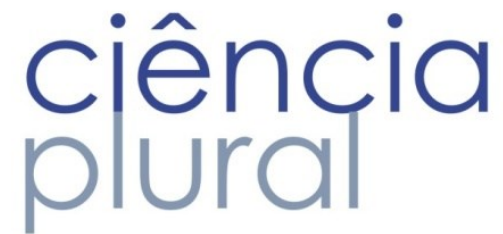

o ideário de equipes da estratégia saúde da família da Grande Florianópolis. Rev. saúde pública. Santa Catarina, 2010; 3(1): 42-56.

7. Ministério da Saúde (Brasil). Resolução n 466, de 12 de dezembro de 2012. Diretrizes e normas regulamentadoras de pesquisas envolvendo seres humanos. Ministério da Saúde/Conselho Nacional de Saúde, Brasília; 2012.

8. Leão CDA, Caldeira AP, Oliveira MMC. Atributos da atenção primária na assistência à saúde da criança: avaliação dos cuidadores. Rev. Bras. Saude Mater Infant. Recife, July-Sept. 2011; 11(3): 14-20.

9. Brandão ALRBS, Giovanella L, Campos CEA. Avaliação da atenção básica pela perspectiva dos usuários: adaptação do instrumento EUROPEP para grandes centros urbanos brasileiros. Ciênc. saúde coletiva. 2013; 18(1): 103-114.

10. Santos I, Alves ACS, Silva AFL, Caldas CP, Berardinelli LMM, Santana RF. O grupo pesquisador construindo ações de autocuidado para o envelhecimento saudável: pesquisa sociopoética. Esc. Anna Nery. Rio de Janeiro, Oct.-Dec. 2011; 15(4): 746-754.

11. Tavares DMS, Dias FA. Capacidade funcional, morbidades e qualidade de vida de idosos. Texto \& contexto enferm. Florianópolis, Jan.-Mar. 2012; 21(1): 112-20.

12. Mazini Filho ML, Zanella AL, Aidar FJS, Silva AMS, Matos DG. Atividade física e envelhecimento humano: a busca pelo envelhecimento saudável. Revista Brasileira de Ciências do Envelhecimento Humano. Passo Fundo, jan-abr. 2010; 7(1): 97-106.

13. Toscano JJO, Oliveira ACC. Qualidade de vida em idosos com distintos níveis de atividade física. Rev. bras. ciênc. esporte. Niterói, May-Jun. 2009; 15(3): 169-173.

14. Silva SF. Organização de redes regionalizadas e integradas de atenção à saúde: desafios do Sistema Único de Saúde. Ciênc. saúde coletiva. Brasil, 2011; 16(6): 2753-62.

15. Escorel S, Giovanella L, Mendonça MHM, Senna MCM. O Programa de Saúde da Família e a construção de um novo modelo para a atenção básica no Brasil. Rev. panam. salud pública. Washington, Feb-Mar. 2007; 21(2-3):164-176. 